\title{
Dependence of bunch energy loss in cavities on beam velocity
}

\author{
Sergey S. Kurennoy \\ LANSCE-1, Los Alamos National Laboratory, Los Alamos, New Mexico 87545
}

(Received 22 December 1998; published 5 March 1999)

\begin{abstract}
Beam energy loss in a cavity can be easily computed for a relativistic bunch using time-domain codes like MAFIA or ABCI. However, for nonrelativistic beams the problem is more complicated because of difficulties with its numerical formulation in the time domain. We calculate the cavity loss factors for a bunch in frequency domain as a function of its velocity and compare results with the relativistic case. [S1098-4402(99)00030-0]
\end{abstract}

PACS numbers: 41.75.-i, 41.20.-q

\section{INTRODUCTION}

Many accelerator facilities, which are now in the design or construction stage such as Accelerator Production of Tritium (APT) [1], Spallation Neutron Source (SNS) [2], and projects aimed toward the accelerator-driven transmutation of nuclear waste (ATW) [3], will use linacs to accelerate intense proton or $\mathrm{H}^{-}$beams providing the final beam power in the range 1-200 MW. Most of them will also rely on superconducting (SC) technology for the accelerating cavities, e.g., [1,3], to reduce operational costs. With the final beam energies around $1-2 \mathrm{GeV}$, the ion beam during acceleration changes its velocity from a nonrelativistic one to $\beta=v / c=0.9-0.95$. At the same time, the number of types of SC cavities is limited to a few due to cost and production reasons. For example, in the APT linac design [1] there will be only two types of SC cavities, optimized for $\beta=0.64$ and $\beta=0.82$, respectively. They will accelerate protons in the velocity range from $\beta=0.58$ (proton energy $211 \mathrm{MeV})$ to $0.94(1.7 \mathrm{GeV})$; at lower energies the normal conducting cavities will be used. Obviously, it is important to know how the amount of the beam energy that is deposited in the cavities and heats them depends on the beam velocity. In other words, one needs to calculate how the beam loss factors depend on $\beta$.

It is common to believe that loss factors of a bunch moving along an accelerator structure at velocity $v=\beta c$ with $\beta<1$ are lower than those for the same bunch in the ultrarelativistic case $\beta \rightarrow 1$. The physical reason for this is that the energy loss should vanish when $\beta \rightarrow 0$. Direct computations with time-domain codes like MAFIA [4] or ABCI [5] are possible only for $\beta=1$. The main difficulty in the numerical time-domain approach for $\beta<1$ is to formulate proper boundary conditions at the open ends of the beam pipe. Instead, the loss factors are computed numerically for the ultrarelativistic bunch, which is a relatively straightforward task, and are considered as upper estimates for the case in question, $\beta<1$. However, it is not obvious that the energy loss depends on the beam velocity monotonously.

The present paper studies $\beta$ dependence of loss factors in more detail. We are primarily concerned about the cavity modes below the beam pipe cutoff frequency, since these modes mostly contribute to the cavity heat load. The frequency-domain approach is applied to calculate the velocity dependence of the bunch energy loss in a few different cases. In general, it would be desirable to develop a simple method for obtaining the answer for the $\beta<1$ beam directly from the results for $\beta=1$. We find, however, that the results for specific cases depend on the properties of the structure, such as the cavity shape and size, as well as on the bunch length. Moreover, the above assumption on the upper estimate from the ultrarelativistic case might be incorrect, especially if only some individual modes of the cavity are concerned.

\section{BEAM COUPLING IMPEDANCES AND LOSS FACTORS OF A CAVITY}

In the frequency domain and in the "closed-cavity" approximation (which means very narrow beam pipes), the beam coupling impedance calculation can be reduced to an internal eigenvalue boundary problem. Since the solution of an inhomogeneous equation can be represented in the form of a series in eigenfunctions (EFs), the impedance is expressed as a formal series. Let $\vec{E}_{s}, \vec{H}_{s}$, where $s=\left(s_{1}, s_{2}, s_{3}\right)$ is a generalized index, be a complete set of EFs for the boundary problem in a closed cavity with perfect walls. The longitudinal impedance is then given by (e.g., [6])

$$
Z(\beta, \omega)=-i \omega \sum_{s} \frac{1}{\omega_{s}^{2}-\omega^{2}} \frac{\left|I_{s}(\beta, \omega)\right|^{2}}{2 W_{s}},
$$

where $I_{s}(\beta, \omega)=\int_{L} d z \exp (-i \omega z / \beta c) E_{s z}(0, z)$ is the overlap integral and $W_{s}$ is the energy stored in the $s$ th mode. Here $E_{s z}(0, z)$ is the longitudinal component of the $s$ th mode electric field taken on the chamber axis.

As seen from Eq. (1), there is a resonant enhancement of the sth term in the series $Z(\beta, \omega)$ at $\omega \rightarrow \omega_{s}$. Let us introduce a finite, but small, absorption into the cavity walls by adding an imaginary part to the eigenvalue: $\omega_{s} \rightarrow \omega_{s}^{\prime}-i \omega_{s}^{\prime \prime}=\omega_{s}^{\prime}\left(1-i / 2 Q_{s}\right)$ [7]. Here, the $Q$ value of the $s$ th mode is $Q_{s}=\omega_{s}^{\prime} W_{s} / P_{s} \gg 1$, where $P_{s}$ 
is the averaged power dissipated in the cavity walls (and/ or, in a real structure, is due to radiation into beam pipes). The wall loss can be expressed as

$$
P_{s}=\frac{1}{2 \sigma_{w} \delta} \int_{S} d s\left|H_{s \tau}\right|^{2},
$$

where $\sigma_{w}$ is the conductivity of the wall material, $\delta$ is the skin depth at the frequency $\omega_{s}^{\prime}, H_{s \tau}$ is the tangential component of the field near the wall, and integration is over the inner cavity surface. For $\omega \simeq \omega_{s}^{\prime}$, the $s$ th term in Eq. (1) for the longitudinal impedance dominates

$$
Z\left(\omega \simeq \omega_{s}^{\prime}\right) \simeq R_{s}=\frac{Q_{s}}{2 \omega_{s}^{\prime} W_{s}}\left|I_{s}\left(\beta, \omega_{s}^{\prime}\right)\right|^{2} ;
$$

or, taking into account the expression for the $Q$ value,

$$
R_{s}(\beta)=\frac{\sigma_{w} \delta\left|\int_{L} d z \exp \left(-i \frac{\omega_{s} z}{\beta c}\right) E_{s z}(0, z)\right|^{2}}{\int_{S} d s\left|H_{s \tau}\right|^{2}} .
$$

The quantity $R_{s}(\beta)$ is the shunt impedance of the $s$ th cavity mode and, unlike the $Q$ factor, it depends on $\beta$.

The bunch loss factor is defined as

$$
k=\frac{1}{\pi} \int_{0}^{\infty} d \omega \operatorname{Re} Z(\beta, \omega)|\lambda(\omega)|^{2},
$$

where $\lambda(\omega)=\int d s \exp [i \omega s /(\beta c)] \lambda(s)$ is a harmonic of the bunch spectrum. For a Gaussian bunch with rms length $2 \sigma$, the line density is $\lambda(s)=\exp \left(-s^{2} / 2 \sigma^{2}\right) /(\sqrt{2 \pi} \sigma)$ and $\lambda(\omega)=\exp \left\{-[\omega \sigma /(\beta c)]^{2} / 2\right\}$. As a result, Eq. (5) takes the form

$$
k(\beta, \sigma)=\frac{1}{\pi} \int_{0}^{\infty} d \omega \operatorname{Re} Z(\beta, \omega) \exp \left[-\left(\frac{\omega \sigma}{\beta c}\right)^{2}\right] .
$$

The loss factor is related to the energy $\Delta U$ lost by the bunch as $\Delta U=k q^{2}$, where $q$ is the bunch charge. As a result, the power deposited by the beam consisting of bunches following through the cavity with the bunch repetition rate $f_{\text {rep }}$ is

$$
P=k q^{2} f_{\text {rep }}=k I^{2} / f_{\text {rep }},
$$

where $I=q f_{\text {rep }}$ is the average beam current. Of course, this equation does not account for possible interactions of the bunches through their wakes.

Assuming all $Q_{s} \gg 1$ and integrating formally Eq. (1) for the $\operatorname{Re} Z(\beta, \omega)$, one can express the loss factor (6) as a series,

$$
k(\beta, \sigma)=\sum_{s} k_{s}(\beta, \sigma),
$$

where the loss factors of individual modes $k_{s}$ are given by

$$
\begin{aligned}
k_{s}(\beta, \sigma) & =\frac{1}{2} \exp \left[-\left(\frac{\omega_{s}^{\prime} \sigma}{\beta c}\right)^{2}\right] \frac{\left|I_{s}\left(\beta, \omega_{s}^{\prime}\right)\right|^{2}}{2 W_{s}} \\
& =\exp \left[-\left(\frac{\omega_{s}^{\prime} \sigma}{\beta c}\right)^{2}\right] \frac{\omega_{s}^{\prime} R_{s}(\beta)}{2 Q_{s}} .
\end{aligned}
$$

From a physical viewpoint, the loss factor (9) of a given mode includes two velocity-related effects. The exponent factor shows that the bunch length $\sigma$ effectively increases to $\sigma / \beta$. The $\beta$ dependence of $R_{s}$ is essentially due to that of the cavity transit-time factor for this resonance mode, as one can see from Eq. (4). Some qualitative guesses about the transit-time factor behavior can be made by considering the effective cavity length that scales as $1 / \beta$, but quantitative conclusions from this observation are readily derived only for resonators with short accelerating gaps.

In principle, Eqs. (8) and (9) give us the dependence of the loss factor on $\beta$. However, one should remember that the answer was obtained in the closed-cavity approximation and can be applied to real problems only when the loss factor is dominated by the lowest resonances, below the pipe cutoff, e.g., for a cavity with narrow beam pipes. Moreover, it is only practical when the number of strong resonances is reasonably small, since Eq. (9) shows that the $\beta$ dependence varies from one resonance to another,

$$
\frac{k_{s}(\beta, \sigma)}{k_{S}(1, \sigma)}=\exp \left[-\left(\frac{\omega_{s}^{\prime} \sigma}{c}\right)^{2} \frac{1}{\beta^{2} \gamma^{2}}\right] \frac{R_{S}(\beta)}{R_{S}(1)},
$$

where $\gamma=1 / \sqrt{1-\beta^{2}}$.

It is obvious from Eq. (10) that for long bunches loss factors will decrease rapidly with $\beta$ decrease, as $\exp \left(-\beta^{-2}\right)$. Indeed, one should expect the lowest resonances at frequencies $\omega_{s}^{\prime} \approx c / d$, where $d$ is a typical transverse size of the cavity. The exponent argument $-(\sigma / d)^{2}$ will have a large negative value for $\sigma \geq d$, and the exponential decrease for small $\beta$ will dominate the impedance ratio. The impedance ratio dependence on $\beta$ is generally more complicated, and we consider a few typical examples below.

\section{EXAMPLES}

\section{A. Cylindrical pill box}

Obtaining explicit expressions for the mode frequencies and impedances of a cylindrical cavity in the limit of a vanishing radius of beam pipes, $b \rightarrow 0$, is rather easy. Let the cavity length be $L$ and its radius be $d$. Then the mode index is $s=(m, n, p)$, where $m$ is the number of radial variations, $p$ the number of longitudinal variations for the $E$ field, and $\mu_{m n}$ is the $n$th zero of the firstkind Bessel function $J_{m}(x)$. The resonance $(m, n, p)$ has the frequency approximately equal to the cavity eigenfrequency, $\omega_{m n p}=\sqrt{\mu_{m n}^{2}+(\pi p d / L)^{2}} c / d$. Then the longitudinal impedance of such a "pill-box cavity" is

$$
\begin{aligned}
R_{0 n p}= & \frac{Z_{0}}{2 \pi \beta^{2}} \frac{L^{3}}{d^{2} \delta} \frac{\mu_{0 n}^{2}}{J_{1}^{2}\left(\mu_{0 n}\right)} \frac{c}{\omega_{0 n p} d} \frac{1}{1+\delta_{p 0}+2 d / L} \\
& \times\left[\left(\frac{\omega_{0 n p} L}{2 \beta c}\right)^{2}-\left(\frac{\pi p}{2}\right)^{2}\right]^{-2}\left\{\begin{array}{c}
\sin ^{2} \\
\cos ^{2}
\end{array}\right\}\left(\frac{\omega_{0 n p} L}{2 \beta c}\right) .
\end{aligned}
$$




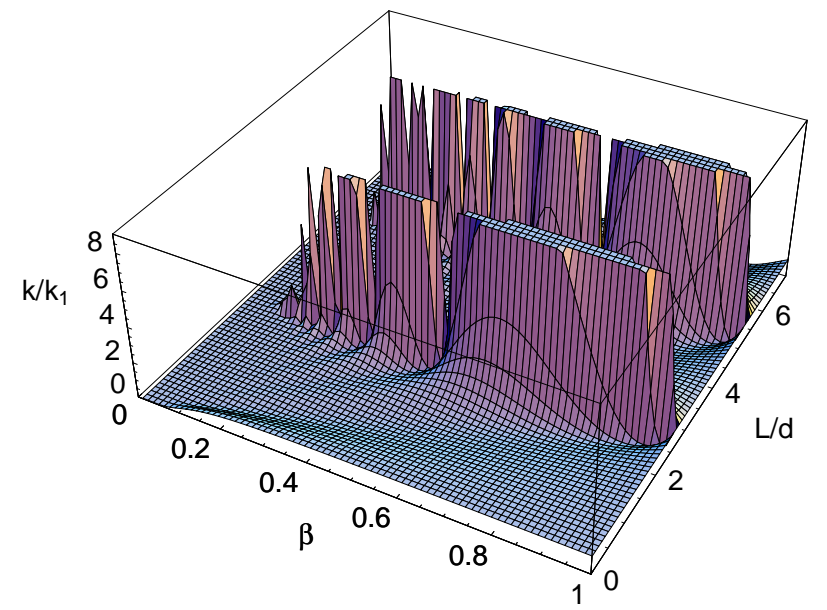

FIG. 1. (Color). The ratio of loss factors for a short bunch, $\sigma / d=0.05$, versus $\beta$ and resonator aspect ratio $L / d$.

The upper line in $\{\cdots\}$ corresponds to even $p$ and the lower one to odd $p$.

The ratio of loss factors Eq. (10) for the lowest $E$ mode, $E_{010}$, is then

$$
\frac{k_{010}(\beta, \sigma)}{k_{010}(1, \sigma)}=\exp \left[-\left(\frac{\mu_{01} \sigma}{d}\right)^{2} \frac{1}{\beta^{2} \gamma^{2}}\right]\left(\beta \frac{\sin \frac{\mu_{01} L}{2 \beta d}}{\sin \frac{\mu_{01} L}{2 d}}\right)^{2}
$$

Obviously, it is almost independent of $\beta$ when the bunch is short, $\sigma \ll d$, and the cavity is short compared to its radius, $L \ll d$. For longer cavities, however, the ratio oscillates and might exceed 1; cf. Fig. 1.

A strong resonance behavior is clearly seen in Fig. 1 for large values of $L / d$, while for small $L / d$ the $k$ ratio slowly decreases with $\beta$ decrease. One should note that, for some particular choices of the parameters, $k_{010}(\beta)$ can be many times larger than $k_{010}(1)$. A picture for a longer bunch will look similar except that the resonances at smaller $\beta$ 's would be damped heavily.

Obviously, a similar behavior is expected for any other individual resonance mode in this case, with peaks at some other values of $\beta$, and of the aspect ratio. Certainly, this example is not practical: we should be interested in the sum of all physically important modes contributing to the bunch energy loss and the cavity heating, say, those below the cutoff. There is no cutoff frequency for the pillbox cavity, and the number of modes to take into account is infinite. Therefore, let us consider some cases with a small number of modes below the pipe cutoff.

\section{B. Small discontinuity}

The simplest case is a small discontinuity on a smooth beam pipe, such as a small axisymmetric cavity or a hole. Let the area of the longitudinal cross section of the cavity be $A$, and its length and depth be small compared to the pipe radius $b$; i.e., $A \ll b^{2}$. It was demonstrated [8] that in this case there exists a trapped

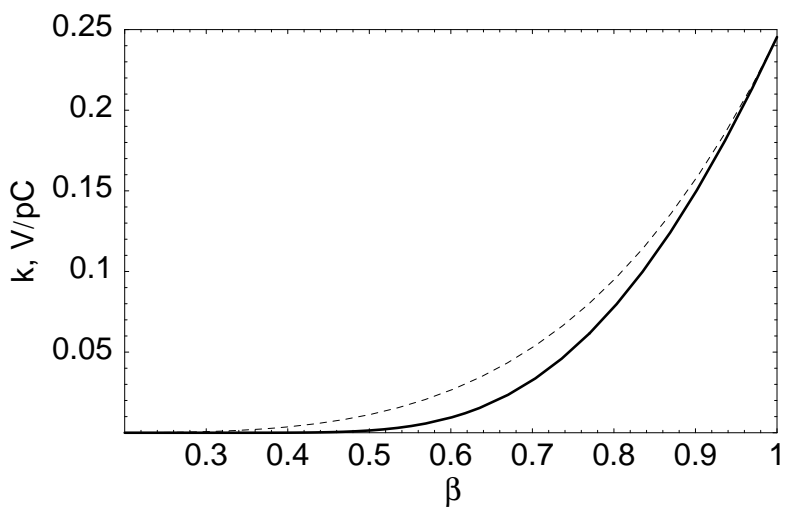

FIG. 2. The loss factor versus $\beta$ for the lowest mode in the pill-box cavity. The dashed line shows the dependence (13) for a small discontinuity.

mode with the frequency slightly below the pipe cutoff frequency $f_{c}=\mu_{01} c /(2 \pi b)$. The on axis longitudinal electric field of the mode is given by a simple expression $E_{z}(z)=E_{z}(0) \exp (-|z| / L)$, where $L=b^{3} /\left(\mu_{01}^{2} A\right) \gg b$ is the characteristic length occupied by the trapped mode in the pipe.

Then the overlap integral in (3) is easily calculated analytically, and the ratio of the shunt impedances Eq. (10) is

$$
\frac{R_{s}(\beta)}{R_{s}(1)}=\beta^{4}\left[\frac{(\omega L / c)^{2}+1}{(\omega L / c)^{2}+\beta^{2}}\right]^{2} \simeq \beta^{4},
$$

where $\omega \simeq 2 \pi f_{c}=\mu_{01} c / b$ is the resonance frequency. The last simplified expression in the right-hand side of Eq. (13) follows from the previous one due to the fact that $\omega L / c \gg 1$, since $\omega b / c \simeq \mu_{01}$ and $L \gg b$. All the above results hold for a small hole in the pipe wall: one just has to substitute $A=\alpha_{m} /(4 \pi b)$, where $\alpha_{m}$ is the magnetic susceptibility of the hole, in all expressions [8].

For larger discontinuities, one can expect some deviation from the simple behavior Eq. (13), even if there is still only one mode below the beam-pipe cutoff. As a simple example, we considered a cylindrical pill-box cavity of depth $h=1 \mathrm{~cm}$ and length $g=2 \mathrm{~cm}$ on the circular pipe of radius $2 \mathrm{~cm}$. Eigenmode calculations with SUPERFISH [9] find a single mode at $f_{r}=4413 \mathrm{MHz}$, below the pipe cutoff frequency. Calculating the overlap integral in Eq. (9) numerically gives us the mode loss factor as a function of $\beta$; see Fig. 2. For comparison, the limiting case of a small discontinuity, Eq. (13), is also presented in Fig. 2. For the ultrarelativistic case, $\beta=1$, the loss factor is $0.24 \mathrm{~V} / \mathrm{pC}$, close to the result for this mode from time-domain calculations performed with $\mathrm{ABCI}$ [5].

\section{APT 1-cell cavity}

As a more realistic example, we consider an APT superconducting 1-cell cavity, e.g., [10]. Of course, such a cavity with wide beam pipes to damp higher order modes cannot be described completely by the formalism 


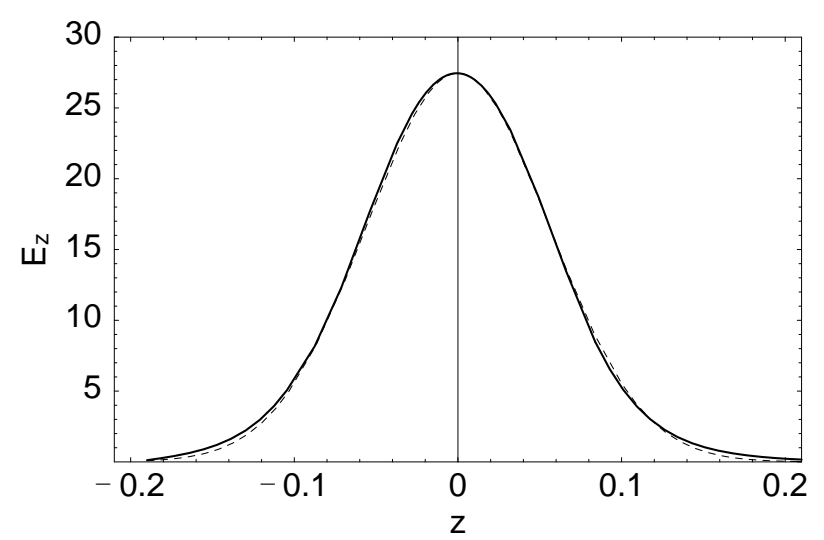

FIG. 3. Longitudinal component of the on axis electric field (arbitrary units) for the fundamental mode in a 1-cell APT $\beta=0.64$ cavity with a power coupler versus $z(\mathrm{~m})$ : MAFIA results (solid line) and analytical fit (dashed line).

of Sec. II, except for the modes below the pipe cutoff. Direct time-domain simulations with the codes MAFIA [4] and $\mathrm{ABCI}[5]$ show the existence of only two longitudinal modes below the cutoff for the $\beta=0.64$ cavity, and only one such mode for $\beta=0.82$, in both cases including the fundamental mode at $f_{0}=700 \mathrm{MHz}$. The loss factor contributions from these lowest resonance modes for a Gaussian bunch with length $\sigma=3.5 \mathrm{~mm}$ for the $\beta=$ 0.64 section, and $\sigma=4.5 \mathrm{~mm}$ for the $\beta=0.82$ section, are about $1 / 3$ of the total loss factor.

We use MAFIA results for the field of the lowest mode (see Fig. 3) to calculate the overlap integral and study the loss factor dependence on $\beta$. The on axis longitudinal field of the fundamental mode is fitted very well by a simple formula $E_{z}(z)=E_{z}(0) \exp \left[-(z / a)^{2}\right]$, where $a=$ $0.079 \mathrm{~m}$ for $\beta=0.64$ and $a=0.10 \mathrm{~m}$ for $\beta=0.82$.

The ratio of the shunt impedances in Eq. (10) is then easy to get analytically,

$$
\frac{R_{s}(\beta)}{R_{s}(1)}=\exp \left[-\frac{1}{2}\left(\frac{\omega a}{c}\right)^{2} \frac{1}{\beta^{2} \gamma^{2}}\right],
$$

where $\omega=2 \pi f_{0}$. The resulting dependence for the $\beta=0.82$ cavity is shown in Fig. 4 , and we see a smooth decrease at lower $\beta$ 's. For $\beta=0.64$, the result is practically identical.

The resulting loss factor for the lowest mode for the cavity design value of $\beta=0.64$ is 0.378 times that with $\beta \rightarrow 1$, and for $\beta=0.82$ it is 0.591 times the corresponding $\beta \rightarrow 1$ loss factor.

\section{APT 5-cell cavities}

For 5-cell APT SC cavities, the lowest resonances are split into five modes which differ by a phase advance per cell, $\Delta \Phi$, and their frequencies are a few percent apart [10]; see below in Tables I and II. The calculated on axis fields of all five modes in the $\mathrm{TM}_{010}$ band, with $\Delta \Phi$ from $\pi / 5$ to $\pi$-the last one is the accelerating mode of the

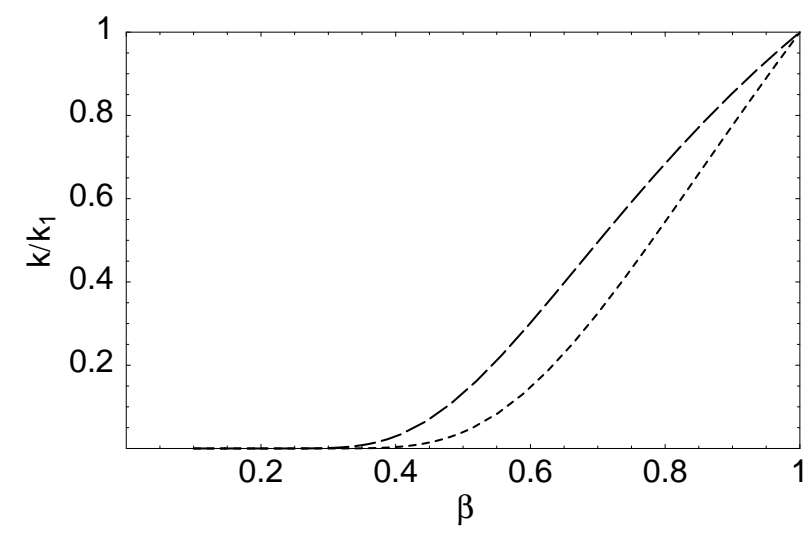

FIG. 4. The loss factor ratio versus $\beta$ for the fundamental mode in 1-cell APT cavities: long-dashed line for $\beta=0.64$ and short-dashed line for $\beta=0.82$.

cavity, are shown for the cavity with $\beta=0.82$ in Fig. 5, left-hand column.

We use MAFIA results for the fields of the modes to calculate overlapping integrals in Eq. (9) with an arbitrary $\beta$ and, therefore, to find their loss factors as a function of $\beta$. The obtained $\beta$ dependencies of the loss factors for the five $\mathrm{TM}_{010}$ modes are shown in the right-hand column of Fig. 5. Obviously, the shunt impedance (and the loss factor) dependence on $\beta$ is strongly influenced by the mode field pattern. The most interesting thing happens, however, when we sum up all five contributions to the loss factor for this band: the resulting dependence on $\beta$ is smooth and the total loss factor decreases monotonically as $\beta$ decreases; see Fig. 6 . Another interesting feature shown in Fig. 6 is that the loss factor of the accelerating mode is maximal near the design $\beta$, while for all other modes it is almost zero in that region. This is not surprising, since the cavity design is optimized for that value of $\beta$ to provide a strong interaction of the accelerating mode with the beam.

A similar picture holds for the $\beta=0.64$ APT cavity: loss factors of individual modes in the given band have a rather irregular $\beta$ dependence, with peaks at different values of $\beta$, but their sum smoothly decreases with the $\beta$ decrease. It works for both bands below the cutoff frequency of the pipe, $\mathrm{TM}_{010}$ and $\mathrm{TM}_{020}$. The total loss factor for all longitudinal modes below the cutoff is shown versus $\beta$ in Fig. 7, as well as the separate contributions of both bands. The contribution of the $\mathrm{TM}_{010}$ band is certainly larger, about $0.5 \mathrm{~V} / \mathrm{pC}$ for $\beta=1$, compared to less than $0.1 \mathrm{~V} / \mathrm{pC}$ from the $\mathrm{TM}_{020}$ band. For the velocity range near the design value of $\beta=0.64, \mathrm{TM}_{010}$ contribution dominates completely. Moreover, in that range the total loss factor is mostly due to the accelerating $\pi$ mode, as one can see in Fig. 7.

Time-domain simulations with the code $\mathrm{ABCI}$ [5] give us the total loss factor of a bunch at $\beta=1$, as well as contributions of separate resonance bands to that loss 
TABLE I. Loss factors (in V/pC) of the lowest modes in the APT 5-cell $\beta=0.64$ cavity.

\begin{tabular}{cccccc}
\hline \hline Mode & $\Delta \Phi$ & $f(\mathrm{MHz})$ & $k(0.64)$ & $k(1)$ & $k(0.64) / k(1)$ \\
\hline \multirow{5}{*}{$\mathrm{TM}_{010}$} & $\pi / 5$ & 682.03 & $2.14 \times 10^{-6}$ & $2.88 \times 10^{-4}$ & $7.4 \times 10^{-3}$ \\
& $2 \pi / 5$ & 687.02 & $2.80 \times 10^{-5}$ & 0.031 & $9.0 \times 10^{-4}$ \\
& $4 \pi / 5$ & 693.16 & $9.05 \times 10^{-6}$ & 0.219 & $4.1 \times 10^{-5}$ \\
& $4 \pi / 5$ & 698.10 & $5.60 \times 10^{-5}$ & 0.251 & $2.2 \times 10^{-4}$ \\
& $\pi$ & 699.96 & 0.186 & $6.76 \times 10^{-3}$ & 27.46 \\
& Total & & 0.186 & 0.507 & 0.366 \\
$\mathrm{TM}_{020}$ & $\pi / 5$ & 1396.82 & $6.45 \times 10^{-4}$ & $5.44 \times 10^{-4}$ & 1.187 \\
& $2 \pi / 5$ & 1410.73 & $1.24 \times 10^{-6}$ & $9.02 \times 10^{-4}$ & $1.4 \times 10^{-3}$ \\
& $3 \pi / 5$ & 1432.74 & $1.83 \times 10^{-5}$ & 0.017 & $1.1 \times 10^{-3}$ \\
& $4 \pi / 5$ & 1458.80 & $8.02 \times 10^{-5}$ & 0.058 & $1.4 \times 10^{-5}$ \\
& $\pi$ & 1481.02 & $3.50 \times 10^{-5}$ & $9.50 \times 10^{-3}$ & $3.7 \times 10^{-5}$ \\
\hline \hline
\end{tabular}

factor. Figure 8 shows the loss factor spectrum for the $\beta=0.64$ APT cavity integrated up to a given frequency versus that frequency. The two lowest steps in Fig. 8, one near $700 \mathrm{MHz}$ with the height $0.5 \mathrm{~V} / \mathrm{pC}$ and the other near $1400 \mathrm{MHz}$ with the height about $0.1 \mathrm{~V} / \mathrm{pC}$, correspond to the two bands of the trapped monopole modes in the cavity, $\mathrm{TM}_{010}$ and $\mathrm{TM}_{020}$. These results agree quite well with our calculations using the frequencydomain approach; cf. Fig. 7 for $\beta=1$ and Table I.

The results for the loss factors of the lowest monopole modes are summarized in Tables I and II. As we mentioned already, the totals for the $\mathrm{TM}_{010}$ and $\mathrm{TM}_{020}$ bands at $\beta=1$ in Table I agree very well with the time-domain results in Fig. 8. Of course, the contribution from higherfrequency modes to the bunch loss factor is also significant, as one can see from Fig. 8. However, the beam energy transferred to the higher modes, which have frequencies above the cutoff and propagate out of the cavity into the beam pipes, will be deposited elsewhere outside the cavity. For the SC cavities, we are mostly concerned about the lowest resonance modes, below the cutoff, since they contribute to the heat load on the cavity itself.

It is worthwhile to note that our results for the design values of $\beta$ are reasonably close to the rough estimates obtained by Krawczyk [10] using MAFIA time-domain simulations with some artificial boundary conditions. The tapered pipes have been introduced at the ends of a rather long structure to reduce reflections caused by improper (at $\beta<1$ ) boundary conditions at the open ends of the beam pipe. After some tricks with the subtraction of wake potentials with and without the cavity, one can get an estimate for the bunch loss factor in that way.

The important observation, however, is that the total loss factors for a given resonance band in Tables I and II are lower for the design $\beta$ than at $\beta=1$. The only exception is the $\mathrm{TM}_{020}$ band in Table II. It, however,

TABLE II. Loss factors (in $\mathrm{V} / \mathrm{pC}$ ) of the lowest modes in the APT 5-cell $\beta=0.82$ cavity.

\begin{tabular}{cccccc}
\hline \hline Mode & $\Delta \Phi$ & $f(\mathrm{MHz})$ & $k(0.82)$ & $k(1)$ & $k(0.82) / k(1)$ \\
\hline \multirow{4}{*}{$\mathrm{TM}_{010}$} & $\pi / 5$ & 674.29 & $9.21 \times 10^{-6}$ & $7.55 \times 10^{-4}$ & 0.012 \\
& $2 \pi / 5$ & 681.11 & $1.43 \times 10^{-4}$ & $2.51 \times 10^{-4}$ & 0.570 \\
& $3 \pi / 5$ & 689.73 & $3.58 \times 10^{-4}$ & 0.029 & 0.012 \\
& $4 \pi / 5$ & 696.87 & $2.94 \times 10^{-4}$ & 0.220 & $1.3 \times 10^{-3}$ \\
& $\pi$ & 699.89 & 0.284 & 0.243 & 1.17 \\
& & & 0.285 & 0.493 & 0.578 \\
$\mathrm{TM}_{020}$ & $\pi / 5$ & 1357.69 & $4.18 \times 10^{-5}$ & $7.98 \times 10^{-7}$ & 52.4 \\
& $2 \pi / 5$ & 1367.65 & $1.36 \times 10^{-4}$ & $8.00 \times 10^{-5}$ & 1.71 \\
& $3 \pi / 5$ & 1384.50 & $1.58 \times 10^{-6}$ & $1.44 \times 10^{-4}$ & 0.011 \\
& $4 \pi / 5{ }^{\mathrm{a}}$ & 1409.56 & $8.04 \times 10^{-7}$ & $1.29 \times 10^{-3}$ & $5.6 \times 10^{-3}$ \\
& $\pi$ & 1436.90 & $1.62 \times 10^{-2}$ & $2.16 \times 10^{-3}$ & 7.50 \\
& & & $1.62 \times 10^{-2}$ & $3.67 \times 10^{-3}$ & 4.41 \\
\hline \hline
\end{tabular}

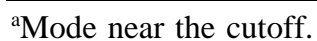

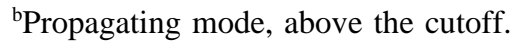



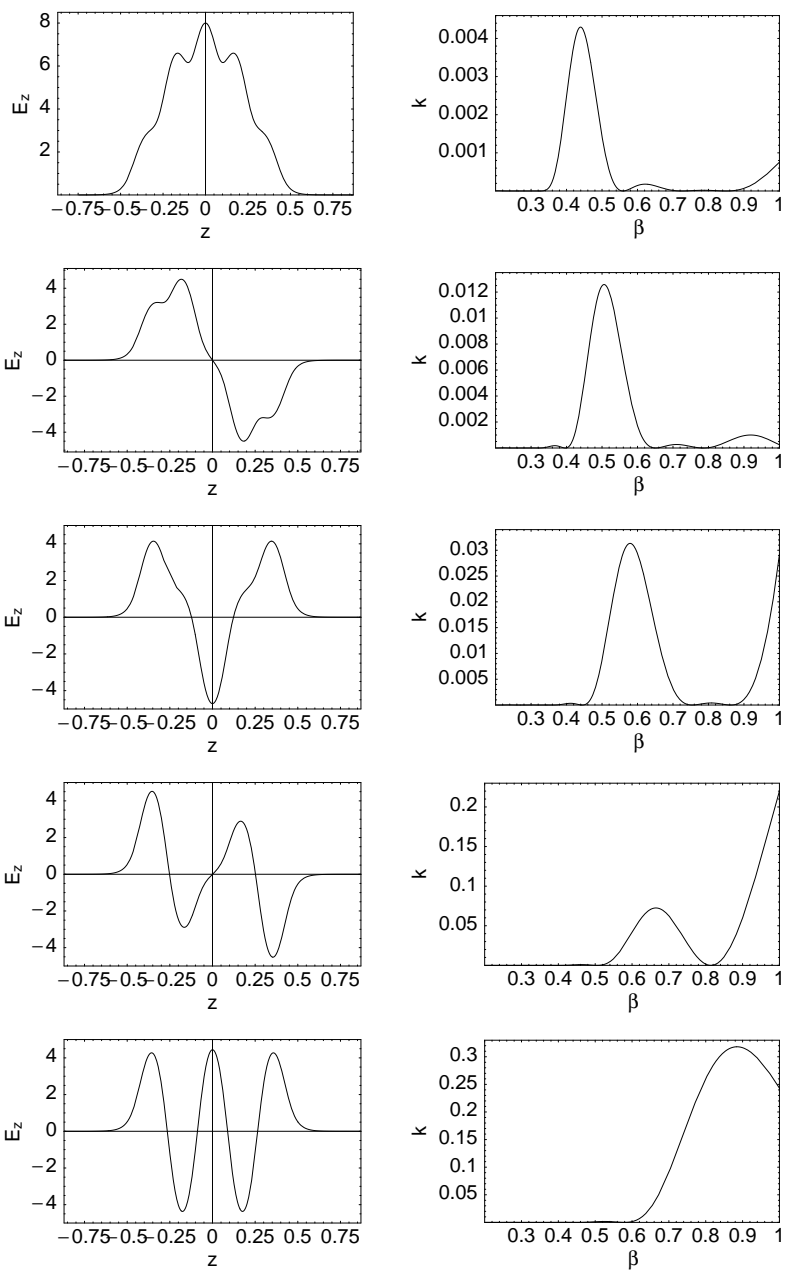

FIG. 5. Longitudinal component of the on axis electric field (arbitrary units) for the $\mathrm{TM}_{010}$ modes in the 5-cell APT $\beta=0.82$ cavity versus $z$ (m) (left-hand column) and their loss factors $(\mathrm{V} / \mathrm{pC})$ versus $\beta$ (right-hand column).

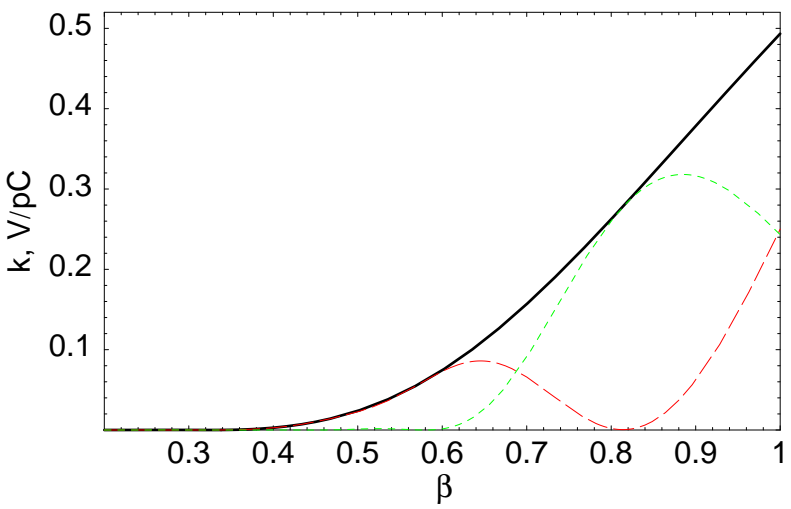

FIG. 6. (Color). The loss factor for all $\mathrm{TM}_{010}$ modes in the 5-cell APT $\beta=0.82$ cavity versus $\beta$ (solid line). The contribution of the accelerating $(\pi)$ mode is plotted by the short-dashed line, and that of all others is plotted by the longdashed line.

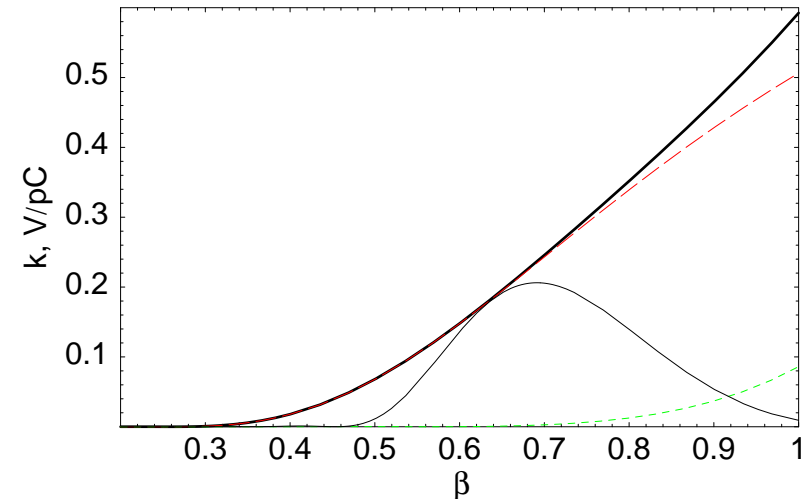

FIG. 7. (Color). The total loss factor for all modes below the cutoff in the 5-cell APT $\beta=0.64$ versus $\beta$. The contribution of the $\mathrm{TM}_{010}$ band is plotted by the long-dashed line, and that of the $\mathrm{TM}_{020}$ band is plotted by the short-dashed line. The thin solid line shows the loss factor for the accelerating mode, $\mathrm{TM}_{010} \pi$ mode.

includes some propagating modes, and, for those, the results of the frequency-domain approach cannot be trusted. Its contribution is certainly very small anyway.

\section{DISCUSSION}

In the examples above, the bunch loss factors were calculated as functions of the beam velocity $\beta<1$ and compared with the corresponding $\beta \rightarrow 1$ results. It has been done by applying the frequency-domain approach rather than the time-domain approach. This approach can be practical when we know the fields of all modes which contribute significantly into the bunch energy loss. In particular, calculating the modes with frequencies above the pipe cutoff presents the most difficulties, since there is no well-established numerical method, except for periodic structures. Nevertheless, for many practical applications,

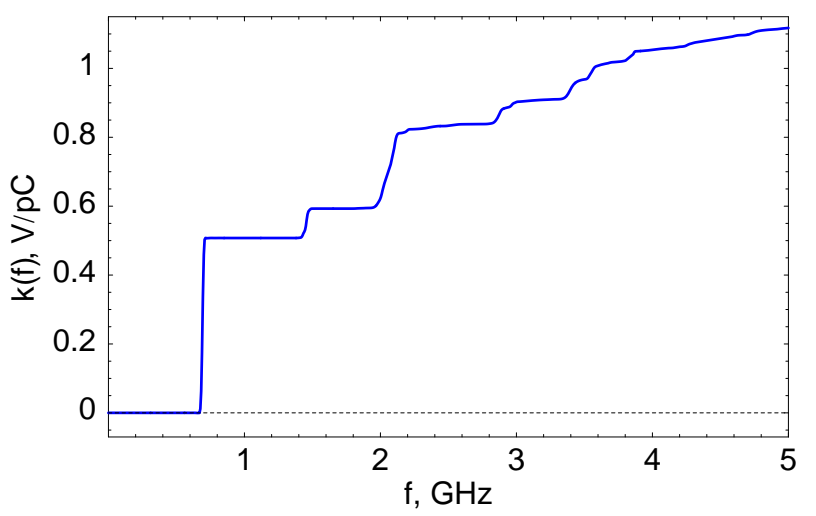

FIG. 8. (Color). Loss factor spectrum integrated up to a given frequency versus that frequency. Results from time-domain simulations with ABCI for a 3.5-mm bunch in the 5-cell APT $\beta=0.64$ cavity. 
especially in superconducting cavities, the contribution of the lowest modes is a major concern, because the abovecutoff modes travel out of the cavity and deposit their energy far away from the structure cold parts, where the heat removal is not a big problem. The method is readily applicable also for cavities with narrow beam pipes.

An interesting observation is that the loss factor of an individual mode at some $\beta<1$ can be larger (and many times larger) than that at $\beta=1$. While not very surprising due to the relation between the loss factor and the transit-time factor discussed in Sec. II, it has an obvious implication that one should exercise caution using $\beta=1$ results as upper estimates for a $\beta<1$ case.

\section{ACKNOWLEDGMENTS}

The author thanks Frank Krawczyk for fruitful discussions and for providing MAFIA results for the 5-cell cavities. Useful discussions with Robert Gluckstern and Thomas Wangler are gratefully acknowledged.
[1] APT Conceptual Design Report No. LA-UR-97-1329, 1997.

[2] NSNS Collaboration, ORNL Report No. NSNS-CDR-2/ V1, 1997.

[3] See, for example, C. D. Bowman et al., Nucl. Instrum. Methods Phys. Res., Sect. A 230, 336 (1992); F. Venneri et al., LANL Report No. LA-UR-98-608, 1998.

[4] R. Klatt and T. Weiland, in Proceedings of the 1986 Linear Accelerator Conference, Stanford, California, 1986 (SLAC, Stanford, CA, 1986), p. 282; MAFIA Release 4.00 (CST, Darmstadt, 1997).

[5] Y.H. Chin, LBL Report No. LBL-35258, 1994.

[6] S. S. Kurennoy, Phys. Part. Nuclei 24, 380 (1993); see also, CERN Report No. SL/91-31 (AP), 1991.

[7] L.D. Landau and I.M. Lifshitz, Electrodynamics of Continuous Media (Pergamon Press, Oxford, 1960).

[8] G. V. Stupakov and S. S. Kurennoy, Phys. Rev. E 49, 794 (1994); S. S. Kurennoy, Phys. Rev. E 51, 2498 (1995).

[9] K. Halbach and R.F. Holsinger, Part. Accel. 7, 213 (1976).

[10] F. L. Krawczyk, in Proceedings of the 1997 Particle Accelerator Conference, Vancouver, Canada, 1997 (IEEE, Piscataway, NJ, 1998), p. 3093; see also, LANL Preprint No. LA-UR-97-1710, 1997. 